\title{
METHODS AND RESULTS FOR THE MSLB NEA BENCHMARK USING SIMTRAN AND RELAP-5
}

\author{
JOSÉ M. ARAGONÉS, * CAROL AHNERT, OSCAR CABELLOS, NURIA GARCÍA-HERRANZ, and VANESSA \\ ARAGONÉS-AHNERT Universidad Politécnica de Madrid, Department of Nuclear Engineering Instituto de Fusión \\ Nuclear, c/ José G. Abascal, 2, 28006 Madrid, Spain
}

The purpose of this paper is first to discuss the methods developed in our three-dimensional pressurized water reactor core dynamics code SIMTRAN and its coupling to the system code REL AP-5 for general transient and safety analysis. Then, we summarize its demonstration application to the Nuclear Energy Agency (NE A) / Organization for Economic Cooperation and Develop- ment (OECD) Benchmark on Main Steam Line Break (MSL B), co-sponsored by the U.S. Nuclear Regulatory Commission (NRC) and other regulatory institutions. In particular, our work has been supported by the Spanish "Consejo de Seguridad Nuclear" (CSN) under a CSN research project.

Our results for the steady states and the guided-core transients, proposed as exercise 2 of the MSLB benchmark, show small deviations from the mean results of all participants, especially in core average parameters. For the full-coupled core-plant transients, exercise 3, a detailed comparison with the University of Purdue-NRC results using PARCS/ REL AP-5, shows quite good agreement in both integral and local parameters, especially for the more extreme return-to-power scenario.

KEYWORDS: NEA benchmark,MSLB transient, coupled neutronkinetics thermal hydraulics

\section{INTRODUCTION}

SIMTRAN is our three-dimensional (3-D) pressur ized water reactor (PWR) core dynamics code, under development and validation for 10 yr (Refs. 1 through 5). It was developed as a single code merge, with data sharing through standard Fortran commons, of our 3-D neutronkinetics (NK) nodal code ${ }^{6}$ SIMULA and the multichannel, with cross flows, thermal-hydraulics (TH) code ${ }^{7}$ COBRA-

IIICMIT-2. Both codes solve the 3-D NK and TH fields with maximum implicitness, using direct and iterative methods for the inversion of the linearized systems.

For the Nuclear Energy Agency (NEA)/Organization for Economic Cooperation and Development (OECD) main steam line break (/MSLB) benchmark, ${ }^{8}$ we have provided results for exercise 2 , the steady states and guided-core transients, using our full SIMTRAN code, which uses COBRA for the 3-D core TH transient solution with given core inlet boundary conditions. For exercise 3, the fullcoupled core-system transients, we have used our reduced SIMTRAN code (without COBRA) coupled with RELAP-5 (Ref. 9), using the same code version and input deck for RELAP-5 as supplied by the University of PurdueU.S. Nuclear Regulatory Commission(NRC) group, which we fully acknowledge. ${ }^{10,11}$ They validated the RELAP-5 system model for exercise 1, while for exercises 2 and 3, they used their own 3-D NK code ${ }^{11}$ PARCS.

Our results, for the steady states and the guided-core transients proposed for exercise 2 of the MSLB benchmark, include a best-estimate scenario, with the physical control rod absorption cross-section sets and a return-topower scenario, with reduced control rod absorption crosssection sets. These cross-section sets were developed by the benchmark coordinators and supplied in its specifications. ${ }^{8}$ Our results show small deviations from the mean results of all participants, especially for core average parameters, while our detailed 3-D results show higher radial and axial power peaks in the all-rods-in-minusone rod stuck-out (ARI-1) and final states, but they show a quite good transient evolution.

For exercise 3, the full-coupled core-plant transients, we present a detailed comparison with the PurdueNRC results using PARCS0RELAP-5 (Refs. 10 and 11), where we have used the same RELAP- 5 version ${ }^{9}$ and input data decks, ${ }^{11}$ but with our SIMTRAN neutronics solution (just as in exercise 2) replacing the PARCS code. The agreement is quite good for both integral and local parameters, especially for the more extreme return-topower scenario.

*E-mail: arago@din.upm.es 


\section{THE SIMTRAN CODE FOR 3-D DYNAMICAL ANALYSIS OF PWR CORES}

\section{II.A. Characteristics of SIMTRAN and the Coupling of Neutronics and TH}

SIMULA is our 3-D nodal NK code for PWR cores. It solves the neutron diffusion equations, in one or two groups, on coarse-mesh nodes (quarters of fuel assemblies) using a linear-discontinuous finite-difference scheme, ${ }^{6}$ where the interface net currents are given in terms of the actual node average and the corrected interface averaged fluxes, using synthetic interface flux discontinuity factors for each group and node interface. SIMULA uses these synthetic coarse-mesh discontinuity factors, in the $X Y$ directions, precalculated by twodimensional (2-D) pin-by-pin two-group diffusion calculations of whole core planes. ${ }^{6}$ In the axial direction it performs embedded iterative one-dimensional (1-D) finemesh two-group diffusion solutions for each node stack, with the radial leakage terms interpolated from the 3-D nodal two-group solution. ${ }^{1-3}$

To solve the NK equations, with six groups of delayed neutron precursors, we use a forward linear time differencing for the six precursor concentration equations, which are implicitly substituted in the fission source nodal equations. The exponential expansion in time of the fission source, with nodal frequencies from the previous time step, and its linear time differencing results in the following nondiagonal linear system:

$$
\begin{aligned}
\left(\sum_{m=1}^{6} W_{n m}\right. & +\frac{1}{k_{\infty}}-1+\frac{\Lambda_{n}}{\Delta t}+\Lambda_{n} \omega_{n} \\
& \left.+\beta_{n} \sum_{d=1}^{6} \frac{f_{d}}{1+\lambda_{d} \Delta t}\right) S_{n}-\sum_{m=1}^{6} W_{m n} S_{m} \\
= & \frac{\Lambda_{n}}{\Delta t} S_{n}^{0} e^{\omega_{n} \Delta t}+\sum_{d=1}^{6} \frac{\lambda_{d} C_{d n}^{0}}{1+\lambda_{d} \Delta t},
\end{aligned}
$$

where

$S_{n}=$ relative fission source of node $n$ at time $t$, with superscript 0 at time $(t-\Delta t)$

$k_{\infty n}=$ infinite multiplication factor (fission source over absorptions) of node $n$

$W_{n m}=$ finite-difference synthetic coefficient, such that $\left(W_{n m} S_{n}-W_{m n} S_{m}\right)$ is the net neutron leakage from node $n$ to node $m$ (Ref. 6)

$\Lambda_{n}=$ mean neutron generation time(s) of node $n$

$\Delta t=$ time step $(\mathrm{s})$

$\omega_{n}=$ exponential frequency $\left(\mathrm{s}^{-1}\right)$ of the fission source at node $n$, given by

$$
\omega_{n}=\frac{1}{\Delta t} \ln \frac{S_{n}(t)}{S_{n}^{0}(t-\Delta t)}
$$

$\beta_{n}=$ effective fraction of delayed neutrons per fission of node $n$

$f_{d}=$ fraction of the delayed neutron precursor group $d(d=1,6)$

$\lambda_{d}=$ half-life $\left(\mathrm{s}^{-1}\right)$ of the delayed neutron precursor group $d$

$C_{d n}^{0}=$ relative nodal concentration of the delayed neutron precursor group $d$ of node $n$ at the previous time step $(t-\Delta t)$.

Note that the extra diagonal terms [fourth to sixth in Eq. (1)] are small and positive, except for large negative frequencies (during rod trips), which are moved to the source term in the right-hand side of the equation. This property preserves the diagonal dominance of the linear system, required for quick convergence of standard iterative methods, such as the tridiagonal inversion on axial lines of nodes with overrelaxed Gauss-Seidel on the $X-Y$ node lines.

The coefficients of the one-group Eq. (1) are iteratively calculated from the embedded two-group 1-D axial fine-mesh diffusion solutions for each node stack. The full two-group 3-D nodal neutron flux solution is directly obtained from the nodal fission sources and the converged fast-to-thermal flux ratios of the 1-D axial solutions. Only a few $(<5)$ outer and $(<20)$ inner iterations are required for convergence at every time step, using an exponential extrapolation of the nodal sources and fluxes to advance the time step and a harmonic interpolation from the axial nodal mesh to the fine mesh (four or eight fine intervals per node, with 34 nodes in the active core).

COBRA-III-C/MIT-2 is a public code ${ }^{7}$ for core TH calculations, with implicit cross flows among channels, and homogeneous two-phase fluids. It is used worldwide for $\mathrm{TH}$ analysis of the departure-from-nucleate-boiling ratio (DNBR) in PWR subchannels, as well as for 3-D whole PWR core simulation with one or more channels per fuel assembly. COBRA uses a direct inversion at each plane of the axial flow equations, with cross flows updated over an outer iteration loop, for the homogenous model single-phase coolant channels, and a finiteelement direct solution of the fuel rod radial temperature equations.

SIMTRAN (Refs. 1 through 5) is our coupled code for 3-D dynamic analysis of PWR cores, integrating our NK code SIMULA (Ref. 6) and the TH code COBRA (Ref. 7) following the scheme of Fig. 1, where the variables exchanged and the external variables driving the transients are shown, together with the correspondence among both NK and TH nodalizations.

The 3-D core NK-TH coupling is done internally in SIMTRAN by a semi-implicit scheme, using a staggered 


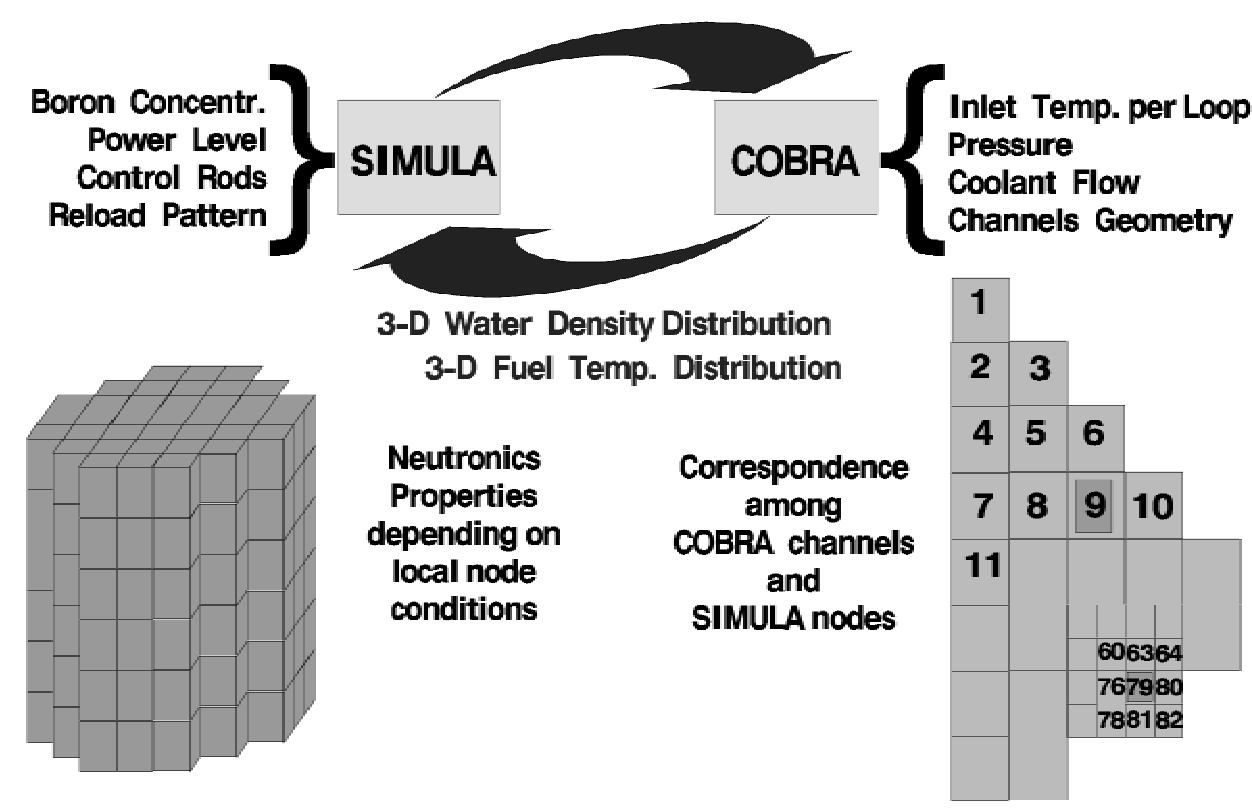

Fig. 1. Variables for the 3-D neutronics TH coupling in SIMTRAN.

alternate time mesh, as shown in Fig. 2. The TH solution is advanced over one-half of the NK time step, thus conserving energy in first order by taking the NK nodal power centered in the time step. Then, the implicitly calculated 3-D TH variables (water density and water and fuel temperatures) are extrapolated over another half of the time step for the NK solution. The neutronics con- stants are thus nearly implicitly calculated in the next time step as a function of the extrapolated $\mathrm{TH}$ variables, where the limited half-step extrapolation prevents significant oscillations, allowing for larger time steps. ${ }^{1,5}$

For the MSLB benchmark, ${ }^{8}$ the SIMTRAN code was extended to deal with axial subdivision of cross-section sets, including varying and moving boundaries, to allow

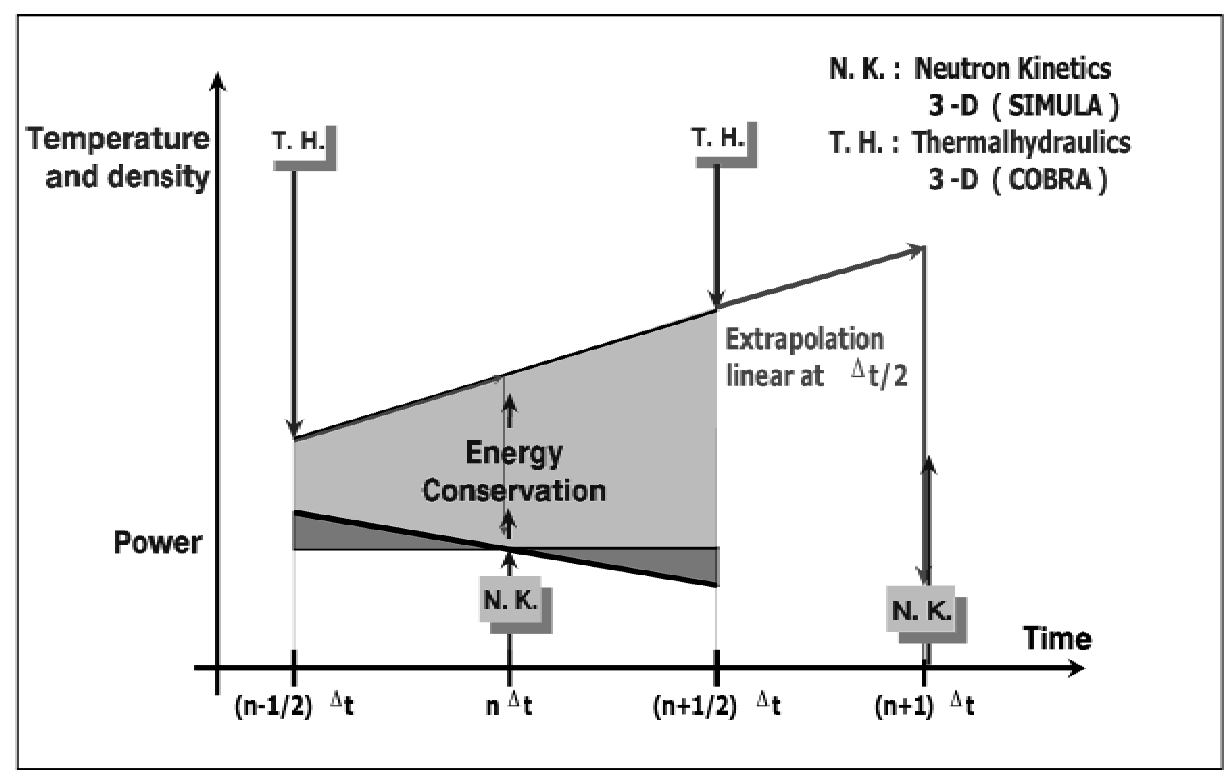

Fig. 2. Temporal coupling of NK and TH for fast transients in SIMTRAN. 
for control rod continuous movement in axially subdivided zones/compositions. The synthetic two-group nodal discontinuity factors were generated by 2 -D finemesh diffusion calculations of the different 15 core planes. This included unrodded and rodded configurations for the initial, midtransient, and final quasi-steady-state conditions, with axial bucklings and local TH conditions per node (quarter of assembly) obtained by iterating the $3-\mathrm{D}$ and 2-D solutions, that converged in two or three iterations. ${ }^{5,12}$

\section{II.B. Special Models: Mixing of Flow from Loops in the Vessel and Subchannel Analysis}

In reactors with multiple loops, it is of special interest to model the water flow and enthalpy mixing from the cold legs inside the reactor vessel (downcomer and bypass) up to the inlet of the core channels, as well as the mixing from the outlet of the core channels in the upper plenum up to the hot leg nozzles, as sketched in Fig. 3. The effect of enthalpy mixing is quite important in the transients with cooling of a single cold loop ${ }^{5}$ or with boron dilution also in a single loop.

SIMTRAN incorporates an empirical model ${ }^{5}$ of the mixing among the cold leg inlets to the vessel to yield the enthalpies at the inlets of the core channels. The model uses Fermi functions for the inlet channel enthalpies in terms of the products enthalpy-mass flow-distance between the vessel inlets of each loop to the core channel inlets, with a single parameter that is fitted to the measurements. The model allows for extreme mass flow and enthalpy variations per loop, as well as for rotational mixing. The same functional model is also used for the mixing from the outlets of the core channels to the vessel

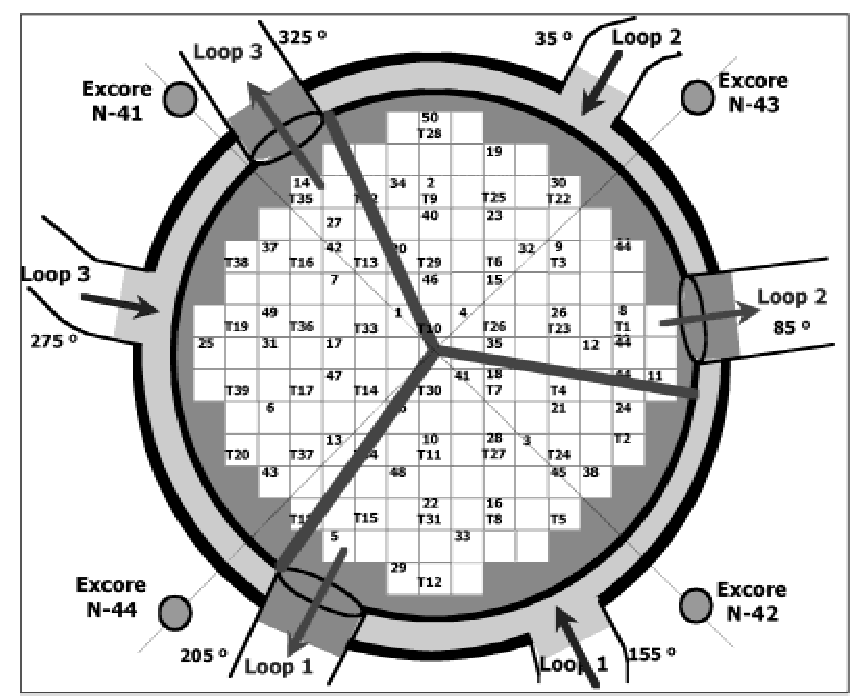

Fig. 3. Mixing of flow enthalpy from loops and effect in the ex-core detectors. outlet nozzles of the hot legs, with a different mixing (exponential) parameter, with larger mixing in the hot upper plenum. ${ }^{5}$

Another special effect to model is that due to the changes in the loop inlet temperatures (cold legs), which cause changes in the water density of the core reflector (downcomer and bypass) and hence in the exponential attenuation of the neutrons that leak from the core through the vessel internals and wall, thus causing the variation of the currents at the ex-core detectors. SIMTRAN uses general response matrices, with exponential attenuation in the reflectors proportional to the water density changes, with each detector affected by the different inlet cold legs, to account for this effect. ${ }^{2-5}$

Another capability developed in SIMTRAN (Refs. 2 through 5) is to perform detailed DNBR analysis in the hottest core subchannels, using 3-D and pin-by-pin powers, by off-line COBRA calculations in one or more subdomains, with a detailed adaptive mesh, as sketched in Fig. 4. Such capability was not required for the MSLB NEA/Nuclear Science Committee (NSC) benchmark.

\section{THE NEA/NSC BENCHMARK ON MSLB IN PWR}

The benchmark on MSLB in PWRs was proposed by the NSC of the NEA/OECD, with the specifications of Ref. 8. It has been carried out along the following coordination workshops:

1. April 1997 in Bethesda (USA), sponsored by the US NRC

2. June 1998 at CIEMAT (Madrid), sponsored by the Spain CSN

3. March 1999 at Garching (Germany), sponsored by the GRS

4. September 1999 at the ETSII-UPM, during MC'99-Madrid

\section{January 2000 at the NEA/OECD (Paris).}

The benchmark is based in the design and operating data of the Three Mile Island Unit 1 PWR, a Babcock \& Wilcox design, at the end of cycle of a recent 24-month operation cycle. The objectives of this benchmark are as follows:

1. verify the capabilities of the TH system codes to analyze complex transients, with coupled 3-D core neutronics and plant interactions

2. in-depth testing of the 3-D neutronics TH coupling

3. evaluate the discrepancies between the predictions of the coupled codes in realistic and bestestimate transient conditions. 


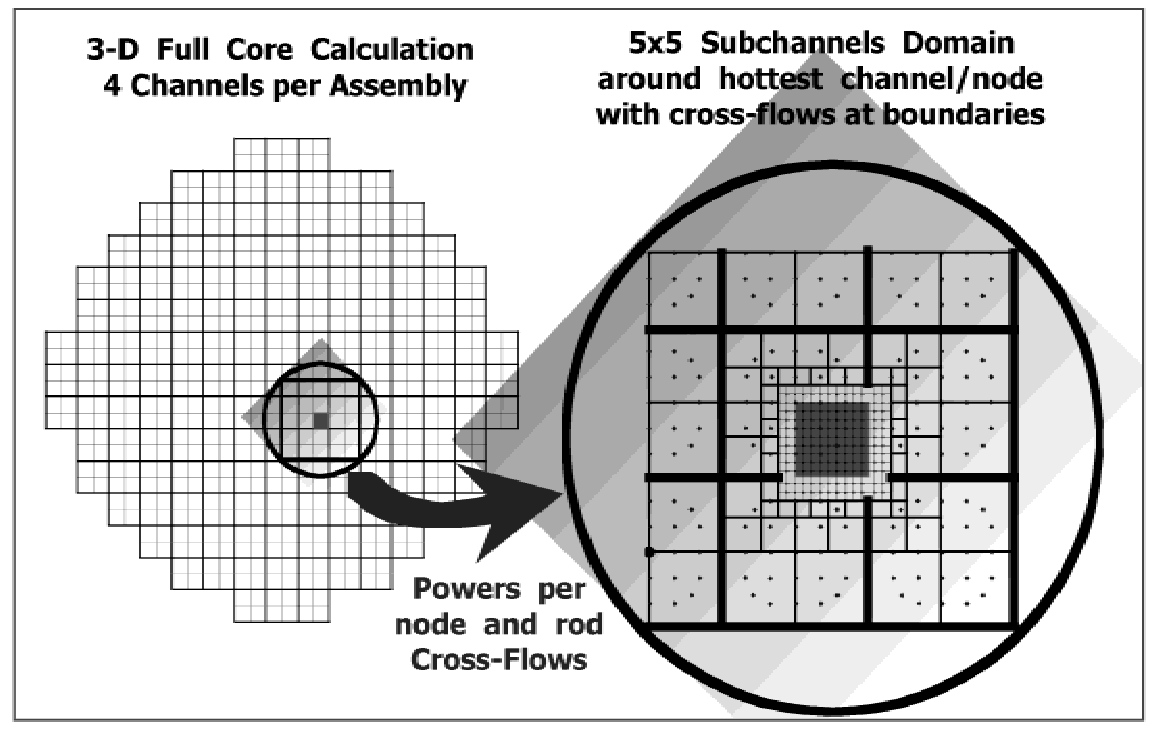

Fig. 4. Analysis of the DNBR per subchannels in subdomains with adaptive mesh.

The phenomenology of the PWR-MSLB transient includes the following main events:

1. guillotine rupture in one main steam line, in one of the two reactor loops

2. loss of mass, depressurization and increase of the steam flow in the secondary side of the affected steam generator

3. cooling of the primary loop, increase of reactivity and core power and trip

4. The most reactive control rod, closer to the cooled loop, is stuck out of the core.

5. The continuous cooling (60 s) can result in return to criticality, or at least in a return to power, for the hypothetical scenario with reduced neutron absorption in control rods.

The MSLB benchmark includes three phases or exercises:

1. Plant simulation, with point kinetics model for the core and the standard TH modeling of the primary and secondary loops: The objective is to verify the response of the TH system models.

2. Coupled 3-D neutronics and TH evaluation of the core response: The objective is to verify the 3-D neutronics core response with imposed $\mathrm{TH}$ core boundary conditions.

3. Full-coupled core-plant best-estimate transient modeling: This exercise simulates the full transient combining the two first phases, verifying in-depth the coupling of the 3-D core NK and the system TH codes.

\section{III.A. Results of the Second Exercise 2 PWR-MSLB: Steady States and Guided Transients}

Exercise 2 includes the calculation of five steady states, as a help to validate the cross-section sets and the 3 -D core neutronics models. The states 0,1 , and 3 are at zero power-it is with all temperatures and densities uniform in the whole core-and with different insertions of the control rod banks: all rods out (ARO), control bank 7 inserted at 900 steps except rod N12 stuck out of core, and all rods inserted at 0 steps except the same rod N12 stuck out too, respectively. Case 4 is identical to case 3 , but with the cross sections for all control rods reduced in their thermal group absorption to allow the hypothetical scenario of return to power. Case 2 is at full power and nominal conditions, with control banks as in case 1.

The results obtained with our SIMTRAN code for all of these steady states are given in Table I, together with the mean values from all the participants in this exercise. ${ }^{8}$ Our results, for the steady states proposed for exercise 2 of the MSLB benchmark, show small deviations from the mean results of all participants, especially for the core average parameters, as is fully documented in the final reports of the benchmark. ${ }^{8}$ Our detailed 3-D results show higher radial and axial power peaks in the ARI-1 and final states. These differences are probably due to the use in our 3-D nodal method of coarse-mesh correction factors synthetically calculated by our standard procedures. ${ }^{5,6,12}$ Our preliminary analysis using a full nonlinear analytical coarse-mesh finitedifference in three dimensions and two groups, recently implemented, ${ }^{13}$ shows a much closer agreement with other advanced nodal codes. 
TABLE I

Results of SIMTRAN and Mean Averages of All Participants for the Steady States*

\begin{tabular}{|c|c|c|c|c|c|c|c|c|}
\hline \multirow[b]{2}{*}{ Case } & \multicolumn{2}{|c|}{$k_{e f f}$} & \multicolumn{2}{|c|}{$F_{\Delta H}$} & \multicolumn{2}{|c|}{$F_{z}$} & \multicolumn{2}{|c|}{ Axial Offset (\%) } \\
\hline & SIMTRAN & Average & SIMTRAN & Average & SIMTRAN & Average & SIMTRAN & Average \\
\hline 0. HZP, ARO & 1.0354 & 1.0337 & 1.353 & 1.361 & 2.709 & 2.684 & +76.5 & +78.0 \\
\hline 1. $\mathrm{HZP}, 90 \%-\mathrm{N} 12$ & 1.0335 & 1.0318 & 1.443 & 1.432 & 2.483 & 2.452 & +71.1 & +71.7 \\
\hline 2. HFP, $90 \%-\mathrm{N} 12$ & 1.0057 & 1.0038 & 1.332 & 1.350 & 1.053 & 1.085 & -0.7 & +3.8 \\
\hline $\begin{array}{l}\text { 3. HZP, ARI-N12 } \\
\text { TRW }(3-1)+\text { SRW }\end{array}$ & $\begin{array}{l}0.9884 \\
4.45 \%\end{array}$ & $\begin{array}{l}0.9854 \\
4.53 \%\end{array}$ & $\begin{array}{l}5.916 \\
0.76 \%\end{array}$ & $\begin{array}{l}5.458 \\
0.70 \%\end{array}$ & 2.816 & 2.754 & +78.4 & +79.1 \\
\hline $\begin{array}{l}\text { 4. HZP, ARI-N12 } \\
\text { TRW }(4-1)+\text { SRW }\end{array}$ & $\begin{array}{l}1.0028 \\
2.99 \%\end{array}$ & $\begin{array}{l}1.0002 \\
3.04 \%\end{array}$ & $\begin{array}{l}3.849 \\
0.43 \%\end{array}$ & $\begin{array}{l}3.630 \\
0.43 \%\end{array}$ & 2.786 & 2.738 & +78.1 & +79.1 \\
\hline
\end{tabular}

${ }^{*} \mathrm{HZP}=$ hot zero power; $\mathrm{ARO}=$ all rods out; $\mathrm{HFP}=$ hot full power; $\mathrm{ARI}=$ all rods in; $\mathrm{TRW}=$ tripped rod worth; $\mathrm{SRW}=\mathrm{stuck}$ rod worth.

In this exercise the transient calculation is limited to the core, in three dimensions and coupled NK-TH, guided by the core boundary conditions, given along the transient by the TRAC code of the benchmark coordinators. ${ }^{8}$ The variables included in the boundary conditions are

1. temperatures at the inlet of the core channels or at the inlet to the vessel of the two loops

2. mass flows at the inlet of the core channels or at the inlet to the vessel per loop

3. pressure at the lower and upper core plena (or as core average).

Exercise 2 of the MSLB benchmark included two guided transients: a best-estimate scenario, with the physical control rod absorption cross-section sets, and a returnto-power scenario, with reduced control rod absorption cross-section sets. The control rod scram is specified at $0.4 \mathrm{~s}$ after reaching the reactor trip setpoint, at $114 \%$ of rated power.

Our results for the return-to-power scenario are given in Figs. 5 and 6 for the evolution of the total core power and the maximum nodal fuel Doppler temperature, together with the results of Purdue/NRC and the mean of all participants in the benchmark.

In exercise 2 the agreement in the evolution of the core power (Fig. 5) is very good since the transients have been guided with specified core inlet boundary conditions. The differences among our SIMTRAN results, using COBRA for core TH, and the Purdue/NRC results, using PARCS and RELAP-5, are minimal. In the maximum nodal fuel temperatures (Doppler average, in Fig. 6), both solutions are in the cluster of solutions supplied by the participants that also use one heat structure (fuel rod) per fuel assembly. For these solutions the maximum fuel temperature is significantly higher at the time of return to power, at $60 \mathrm{~s}$, than for other solutions where the heat structures include several fuel assemblies, thus lowering the mean value.

\section{III.B. Coupling of SIMTRAN to RELAP-5 and TRAC-M}

In the frame of the project for a consolidated $\mathrm{TH}$ code of the NRC, T. Downar and his group at the University of Purdue have developed and distributed ${ }^{10}$ a general interface (GI) to couple their 3-D nodal NK code PARCS to the TH system codes RELAP-5 (Ref. 9) and TRAC-M (Refs. 10 and 11).

Sets of similar routines have been implemented in these codes to perform the data transformations between the different meshes (data mapping) of the respective codes, with their own locations in memory. This is done ${ }^{10}$ by using vectors that group all the data to be exchanged and are transmitted among the codes via the standard Parallel Virtual Machine (PVM) library, developed at Oak Ridge National Laboratory by J. Dongarra ${ }^{14}$ An intermediate program for GI gets these vectors, as well as the semaphores of communication and error, multiplies them by the permutation matrices for the mesh transformation, and sends the product vectors to the other code, also via calls to PVM routines. Figure 7 shows this coupling scheme.

This coupling scheme was designed and implemented with emphasis on the maximum flexibility and minimum modifications in the existing codes, to facilitate their maintenance and portability to other neutronics and $\mathrm{TH}$ codes.

For its implementation in our SIMTRAN code, we have adapted the set of routines named PARCS Data Mapping Routines that perform the mapping of the data to transmit and acquire, to or from the memory layout of PARCS or SIMTRAN and the exchange vectors, and the calls to PVM routines, just replacing the data in the PARCS memory layout (commons) by the corresponding data in the SIMTRAN commons, by a direct mesh transform among both 3-D nodal meshes. 


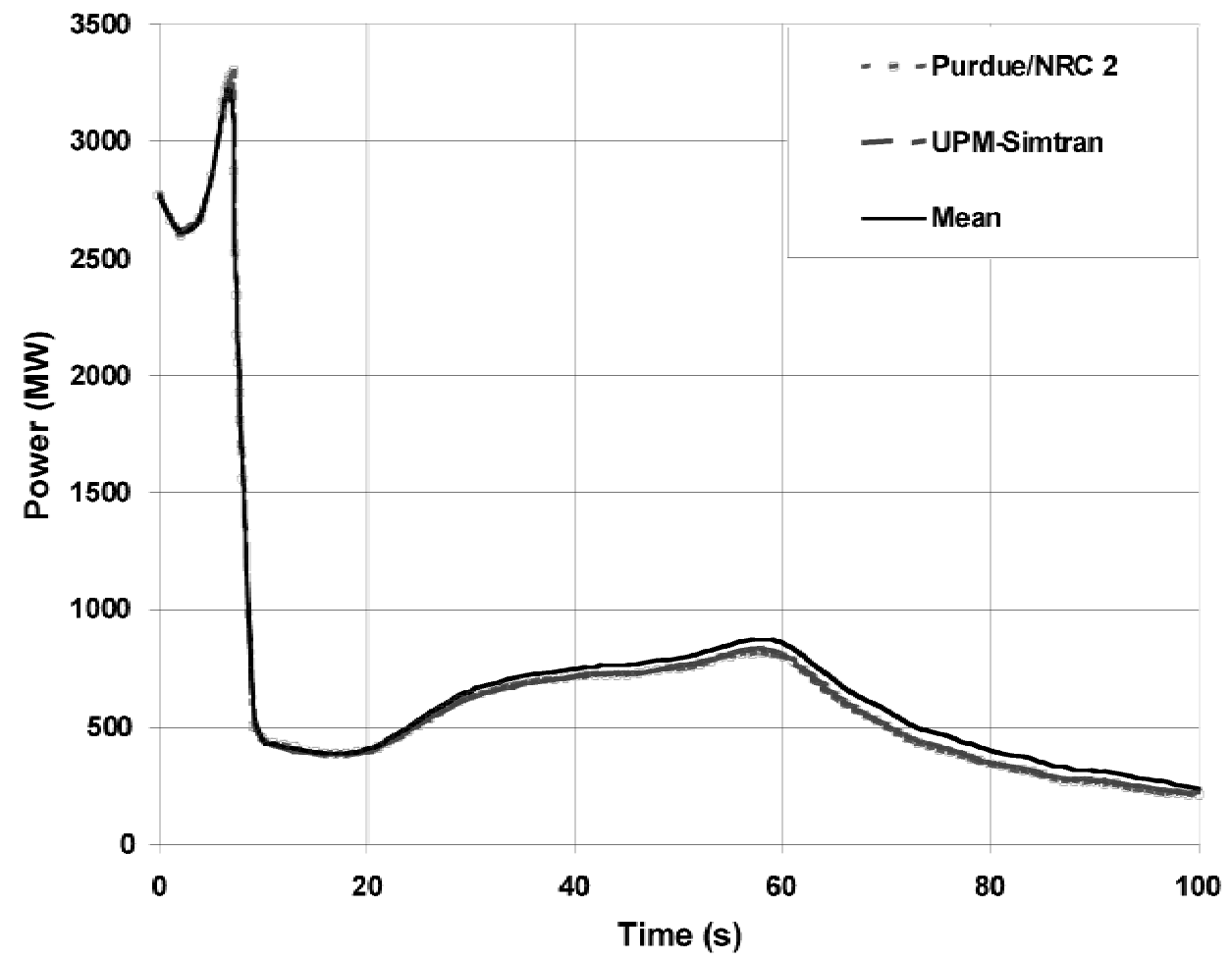

Fig. 5. Total core power versus time in the guided MSLB transient with return to power. ${ }^{\mathrm{a}}$

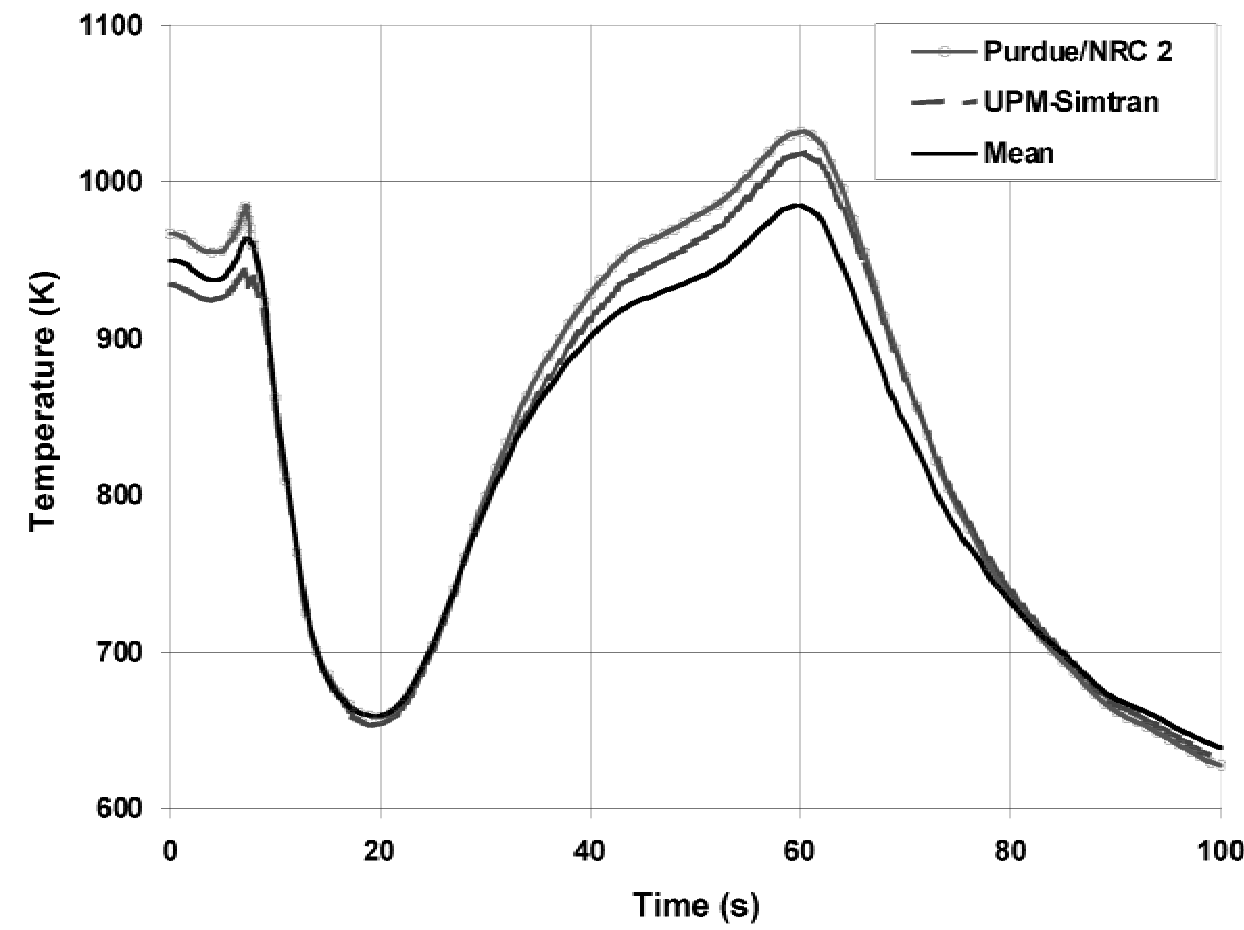

Fig. 6. Maximum nodal temperature in the guided MSLB transient with return to power. ${ }^{\mathrm{a}}$

${ }^{a}$ The Purdue/NRC 2 solution was given by PARCS coupled to RELAP-5 in parallel channels. ${ }^{8,11}$ The UPM-SIMTRAN solution is by our SIMTRAN code, using COBRA with one channel and fuel rod per fuel assembly. ${ }^{8,13}$ The mean curve is the average of the solutions from all participants. ${ }^{8}$ 


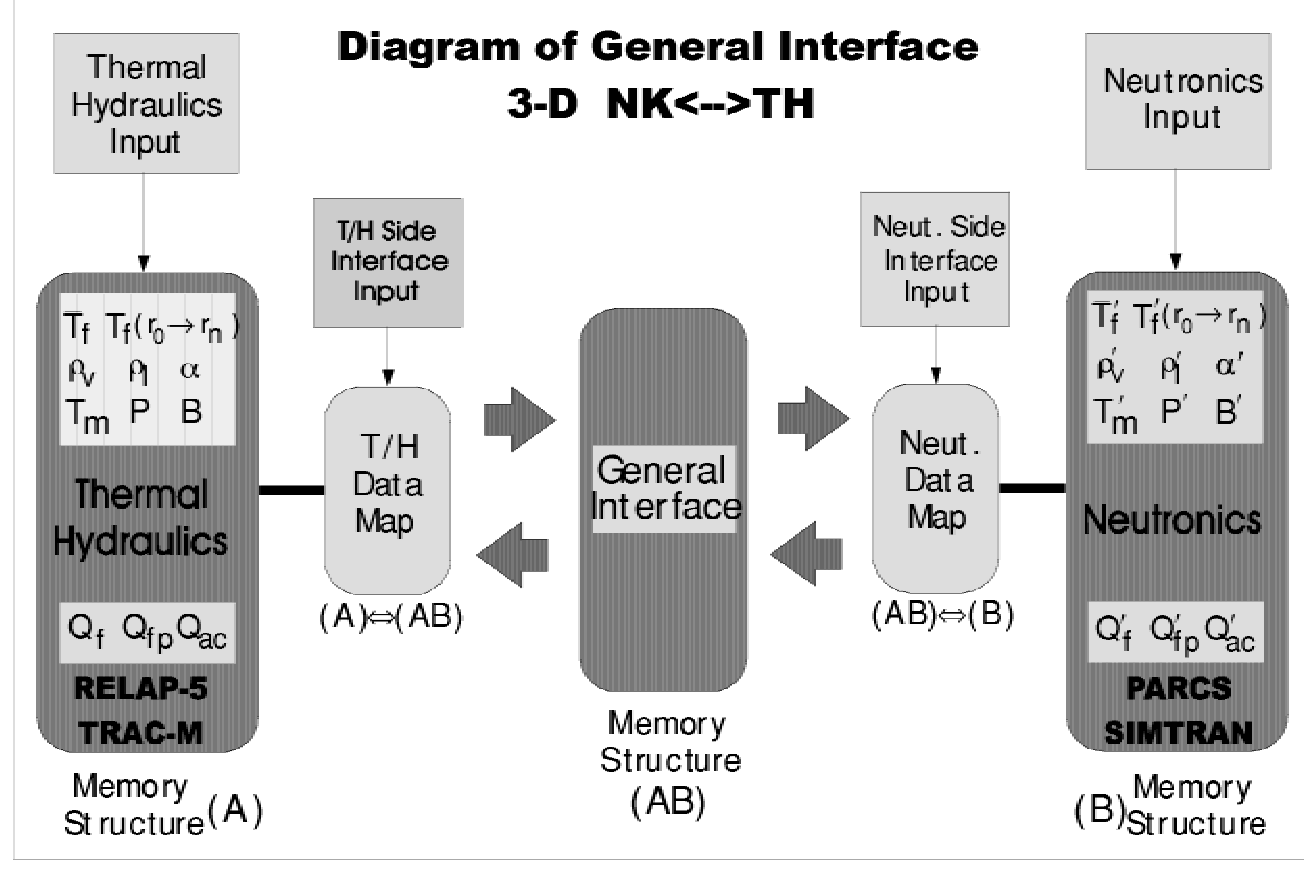

Fig. 7. Scheme of the coupling between PARCS or SIMTRAN and RELAP-5 or TRAC-M.

In this way the permutation matrices for the GI are just the same ones generated to couple PARCS with RELAP-5 or TRAC-M (Refs. 10 and 11), simplifying the management of the databases for validation and applications.

In the implementation in SIMTRAN, we have used our semi-implicit scheme for time coupling in a staggered mesh, described in Sec. II.B and Fig. 2. In the present version, named SIMREL, all the COBRA routines have been suppressed, acquiring instead all the TH variables from the system code RELAP-5.

In the distributed data decks for RELAP-5 (Ref. 11), the number of hydraulics channels parallel or coupled in the core and reflectors is limited to 18 and 1, respectively, for a reduced and practical nodalization that is similar to the one used in the guided-transient (exercise 2) of the MSLB benchmark, while the number of heat structures that are used is much larger, with one mean fuel rod for each fuel assembly. In future work, we plan to reinsert the COBRA code for a more detailed TH core modeling, using one mean hydraulic channel and fuel rod per assembly or quarter of assembly. This requires special procedures to preserve the consistency in the variables of both TH codes (COBRA and RELAP-5) at the core inlet and outlet.

\section{III.C. Results for the Exercise 3 PWR-MSLB: Coupled Plant and 3-D Core Transients}

We have applied SIMTRAN in its version SIMREL interfaced with RELAP-5 to the transients of exercise 3 of the PWR-MSLB benchmark in both best-estimate and return-to-power scenarios. The neutronics databases of SIMTRAN are identical to the ones developed and validated in the exercise 2 (Ref. 12), generated as described above. The TH databases of RELAP-5 are identical to the ones developed and validated by the Purdue-NRC group in exercises 1 and 3 of the benchmark, ${ }^{11}$ where the whole plant is modeled.

In Tables II, III, and IV and Figs. 8, 9, and 10, we collect our results obtained with SIMTRAN coupled to RELAP-5, together with the results obtained by the Purdue-NRC group with PARCS coupled to RELAP-5, to verify the agreement of our code implementation. The time step used is the same for all cases, a fixed step of $0.01 \mathrm{~s}$ given by RELAP-5, with a nodalization that allows this time step without violating the Courant limit along the transients.

Table II includes the sequence of events and times of occurrence for both MSLB transient scenarios, with both code systems. For both scenarios, SIMTRAN/ RELAP-5 shows a delay of a few hundredths of second with respect to PARCS/RELAP-5, probably due to the differences in their time coupling methods, semi-implicit in our case and explicit in the other case.

The results in the core integral parameters (reactivity or power level) and the power distributions at characteristic times of the transient (radial and axial peaking factors and axial offset of power) also are very close for both code systems. Table III includes these parameters at the initial steady state and at the time of maximum power 
TABLE II

Sequence of Events*

\begin{tabular}{|l|c|c|r|r|}
\hline \multirow{2}{*}{ Description of Event } & \multicolumn{2}{|c|}{ Best-Estimate Scenario } & \multicolumn{2}{c|}{ Return-to-Power Scenario } \\
\cline { 2 - 5 } & SIMTRAN + R5 & PARCS + R5 & SIMTRAN + R5 & PARCS + R5 \\
\hline Rupture in main steam line & 0.01 & 0.01 & 0.01 & 0.01 \\
Trip of control rods & 6.18 & 6.17 & 6.18 & 6.17 \\
Closure of turbine valves & 6.68 & 6.67 & 36.68 & 35.94 \\
Injection at high pressure & 35.90 & 35.80 & 65.83 & 65.82 \\
Maximum return to power & 69.70 & 100.00 & 100.00 & 100.00 \\
End of transient & 100.00 & & & \\
\hline
\end{tabular}

*Units of measure are in seconds.

\section{TABLE III}

Initial Steady State at Full Power and at the Time of Maximum Power Before Trip

\begin{tabular}{|l|c|c|}
\hline \multicolumn{1}{|c|}{ Parameter } & $\begin{array}{c}\text { SIMTRAN } \\
+\mathrm{R} 5\end{array}$ & $\begin{array}{c}\text { PARCS } \\
+\mathrm{R} 5\end{array}$ \\
\hline Power at initial state $(\%)$ & 100 & 100 \\
Multiplication factor $\left(k_{e f f}\right)$ & 1.00479 & 1.00528 \\
Radial assembly power factor $\left(F_{x y}\right)$ & 1.326 & 1.332 \\
Axial core power factor $\left(F_{z}\right)$ & 1.062 & 1.070 \\
Axial offset of core power $(\%)$ & -0.39 & +0.47 \\
Power just before trip $(\%)$ & 118.61 & 118.12 \\
Radial assembly power factor $\left(F_{x y}\right)$ & 1.460 & 1.464 \\
Axial core power factor $\left(F_{z}\right)$ & 1.091 & 1.072 \\
Axial offset of core power $(\%)$ & -2.72 & -1.82 \\
\hline
\end{tabular}

before the reactor trip, up to the point where both scenarios show the same behavior.

The agreement of both codes is quite good for these safety-related parameters since the differences are well within the standard acceptance criteria used in nuclear design. Table IV includes the same parameters from the snap-shots at the times of maximum return to power and transient end.

Figure 8 plots the evolution of the dynamical reactivity along the transient as calculated by both code systems for the two scenarios.

The best-estimate scenario, with realistic cross sections of the control rods, is far from a second criticality with both codes. In the return-to-power scenario, with reduced absorption in the control rod cross sections, we obtain a slight second criticality with the SIMTRAN code. The evolution of the reactivity calculated by SIMTRAN and PARCS is very close in both scenarios but with systematic differences, due probably to the way of calculating the dynamical reactivity, by inverse point kinetics in SIMTRAN and by the perturbation method, with separated effects, in PARCS.

Figure 9 plots the evolution along the transients of the total core power for both code systems, with very good agreement, and the core fission power calculated by SIMTRAN, where the residual decay heat is calculated following the benchmark specifications: the initial

TABLE IV

Snapshots at Maximum Return to Power and End of Transient

\begin{tabular}{|l|c|c|c|c|}
\hline \multirow{2}{*}{\multicolumn{1}{|c|}{ Parameter }} & \multicolumn{2}{|c|}{ Best-Estimate Scenario } & \multicolumn{2}{c|}{ Return-to-Power Scenario } \\
\cline { 2 - 5 } & SIMTRAN + R5 & PARCS + R5 & SIMTRAN + R5 & PARCS + R5 \\
\hline Maximum power (\%) & 9.53 & 9.53 & 37.09 & 36.65 \\
Radial $F_{x y}$ & 4.328 & 4.171 & 3.693 & 3.702 \\
Axial $F_{z}(\%)$ & 1.927 & 1.876 & 1.826 & 1.847 \\
Axial offset (\%) & +37.25 & +35.95 & +36.30 & +38.0 \\
Final power (\%) & 4.74 & 4.73 & 2.95 & 8.64 \\
Radial $F_{x y}$ & 2.161 & 1.123 & 1.741 & 1.737 \\
Axial $F_{z}$ & 1.334 & +1.317 & +31.54 & +34.24 \\
Axial offset (\%) & +11.94 & & \\
\hline
\end{tabular}


(\%)

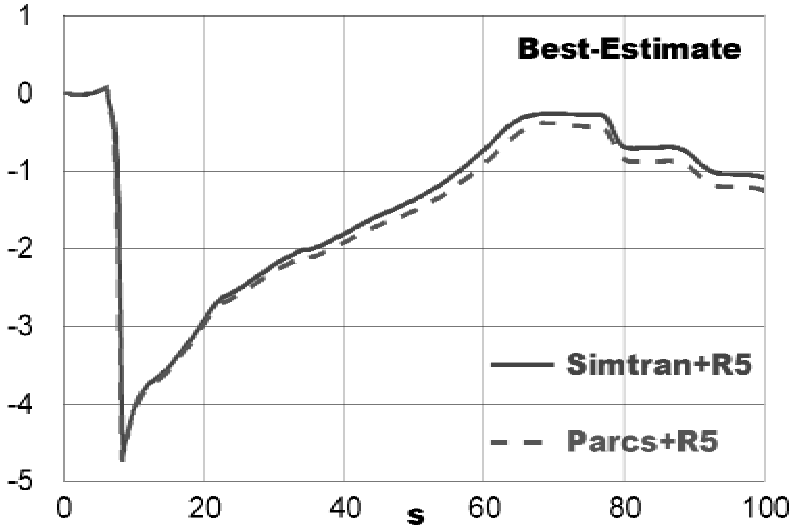

(\%)

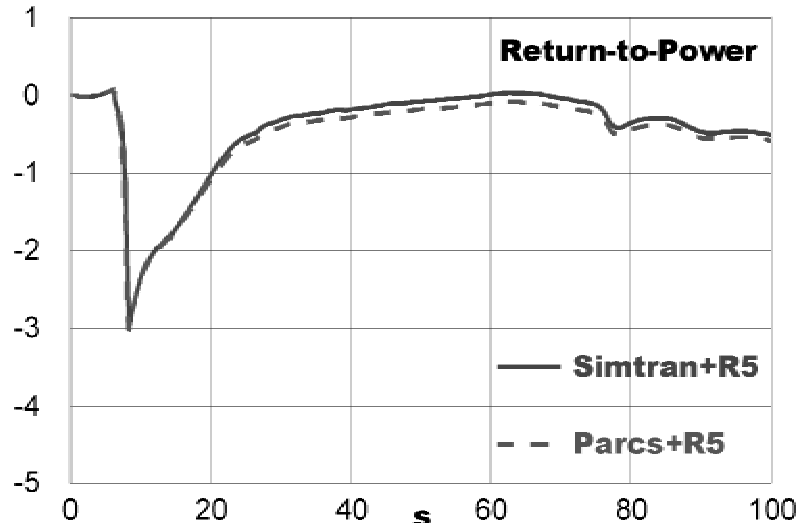

Fig. 8. Dynamical reactivity evolution with SIMTRAN + RELAP-5 and PARCS + RELAP-5.
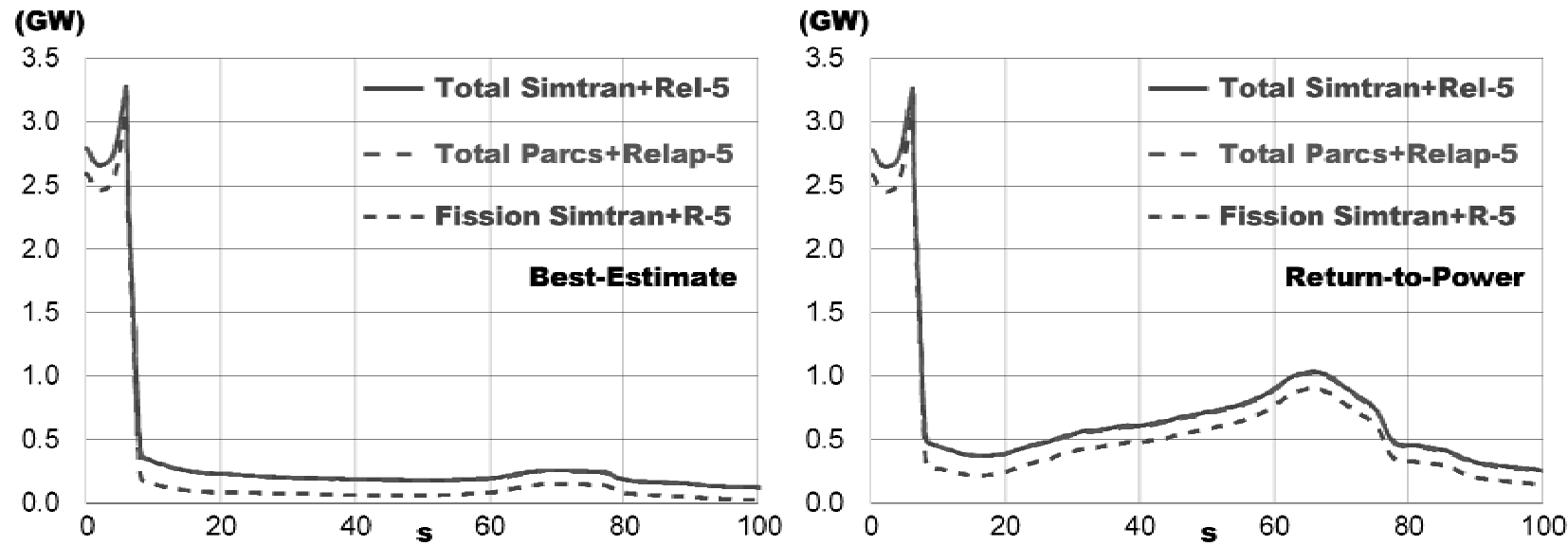

Fig. 9. Core total and fission powers with SIMTRAN + RELAP-5 and PARCS + RELAP-5.

(K)

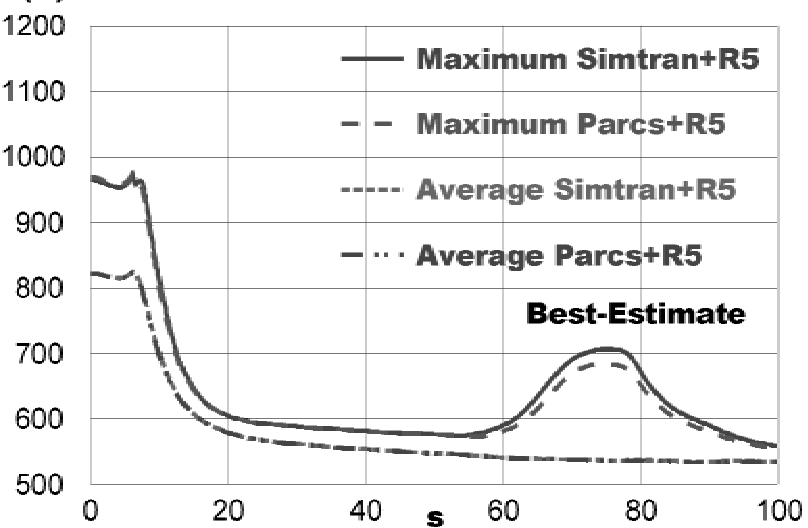

(K)

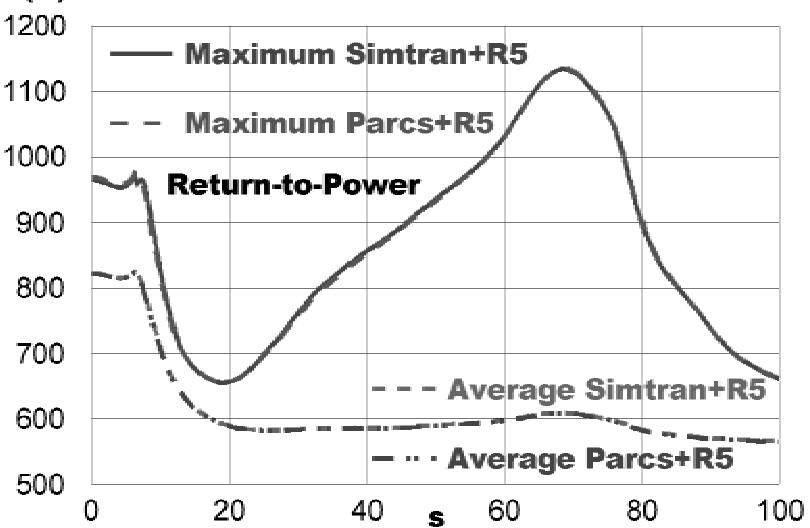

Fig. 10. Core average and maximum nodal fuel temperatures in MSLB transients with SIMTRAN + RELAP-5 and PARCS + RELAP-5. 
3-D shape as a fraction of the total power is just multiplied afterward by the decay heat power given as tables in the benchmark specifications for both scenarios.

As shown in Fig. 10, the agreement in the maximum nodal fuel temperatures (Doppler) is very good for the return-to-power case, as in the core average Doppler temperature for both scenarios. In the best-estimate case a slightly higher difference is observed at the second maximum.

We observe in both codes an increase of $\sim 20 \%$ of the maximum fuel temperature, with respect to the initial, due to the use of one mean fuel rod as a heat structure in RELAP-5 per fuel assembly, and remarkable agreement between both codes in the maximum fuel temperature, for the extreme return-to-power scenario.

\section{CONCLUSIONS AND ONGOING WORK}

We have successfully validated our SIMTRAN code in the NEA/NSC benchmark of the PWR MSLB (Ref. 8), both for core steady state and transient analysis and for whole system transient analysis, coupled to the RELAP-5 code. The differences in the core averages and 3-D distributions of the most relevant parameters are well within the acceptance criteria of nuclear design and safety analysis. This validation is consistent with our previous SIMTRAN results for the NEA/OECD rod ejection and bank withdrawal benchmarks in PWRs (Refs. 1, 3, 5, and 12), as well as our extensive validation against PWR core operating data. ${ }^{2,4,12}$ Validation of the TH system RELAP-5 code was out of the scope of our work since we just adopted to interface our SIMTRAN code with this widely used TH system code to rely upon the bulk of validation work done by others elsewhere.

Our ongoing work follows two main lines: first, to improve the accuracy and efficiency of our NK nodal code, using a full nonlinear analytical coarse-mesh finitedifference nodal method in three dimensions and two groups recently developed and demonstrated, ${ }^{13}$ and second, to improve the accuracy of the reduction of the TH subchannel models to the assembly or multiassembly average channel models, including extended correlations for the channel-averaged nonlinear void fractions and/or coolant densities and effective Doppler fuel temperatures, as well as the extensions of the general and consistent coupling with RELAP-5 and other TH system and core codes, such as TRAC-M and COBRA-TF.

\section{REFERENCES}

1. F. MERINO, C. AHNERT, and J. M. ARAGONÉS, "Development and Validation of the 3-D PWR Core Dynamics SIMTRAN Code," Mathematical Methods and Supercomputing in Nuclear Applications, H. KUSTERS, Ed., Vol. 1, p. 646, Kernforschungzentrum Karlsruhe, Germany (1993).
2. J. M. ARAGONÉS and C. AHNERT, "Computational Methods and Implementation of the 3-D PWR Core Dynamics SIMTRAN Code for Online Surveillance and Prediction," Mathematics and Computations, Reactor Physics and Environmental Analysis, L. BRIGGS, Ed., Vol. I, p. 237, American Nuclear Society (1995).

3. J. M. ARAGONÉS, C. AHNERT, and O. CABELlOS, "Methods and Performance of the 3-D PWR Core Dynamics SIMTRAN Online Code," Nucl. Sci. Eng., 124, 111 (1996).

4. J. M. ARAGONÉS, C. AHNERT, D. CANO, and N. GARCÍA-HERRANZ, "Planning of Operational Maneuvers with the 3-D PWR Core Dynamics SIMTRAN Online Code," Proc. Int. Conf. Physics of Reactors (PHYSOR 96), Mito, Ibaraki, Japan, Vol. 4, K-9-17 (1996).

5. J. M. ARAGONÉS, C. AHNERT, and V. ARAGONÉSAHNERT, "Coupled 3-D Neutronic-Thermalhydraulic Analysis of Transients in PWR Cores," Mathematical Methods and Supercomputing for Nuclear Applications, F. BROWN, Ed., Vol. 2, p. 1380, American Nuclear Society (1997).

6. J. M. ARAGONÉS and C. AHNERT, "Linear-Discontinuous Finite-Difference Formulation for Synthetic Coarse-Mesh FewGroup Diffusion Calculations," Nucl. Sci. Eng., 94, 309 (1986).

7. J. W. JACKSON and N. E. TODREAS, "COBRA IIIc/ MIT-2: A Computer Program for Steady State and Transient Thermo-Hydraulic Analysis of Rod Bundle Nuclear Fuel Elements," Massachusetts Institute of Technology, Boston, Massachusetts (1981).

8. K. N. IVANOV, T. M. BEAM, A. J. BARATTA, A. IRANI, and N. TRIKOUROS, "Pressurized Water Reactor Main Steam Line Break (MSLB) Benchmark," Vol. I, Final Specifications, NEA/NSC/DOC 8 (99); Vol. II, Summary Results of Phase I on Point Kinetics, NEA/NSC/DOC 21 (2000); Vol. III, Results of Phase II on 3-D Core Boundary Conditions Model, NEA/NSC/DOC 12 (2002); Vol. IV, Summary Results of Phase III on Coupled Core-Plant Transient Modelling, NEA/NSC/ DOC, 21 (2003).

9. V. H. RANSON, "Relap5/Mod2 Code Manual,”NUREG/ CR-4312, U.S. Nuclear Regulatory Commission (1985).

10. D. BARBER, R. MILLER, H. JOO, T. DOWNAR, W. WANG, V. MOUSSEAU, and D. EBERT, "Coupled 3-D Reactor Kinetics and Thermal-Hydraulic Code Activities at the US Nuclear Regulatory Commission," Mathematics and Computation, Reactor Physics and Environmental Analysis in $\mathrm{Nu}$ clear Applications, J. M. ARAGONÉS, Ed., Vol. 1, p. 311, Senda Editorial, Madrid, Spain (1999).

11. R. MILLER, H. JOO, D. BARBER, T. DOWNAR, and D. EBERT, "Analysis of the OECD MSLB Benchmark with RELAP-PARCS and TRAC-M-PARCS," Mathematics and Computation, Reactor Physics and Environmental Analysis in Nuclear Applications, J. M. ARAGONÉS, Ed., Vol. 1, p. 321, Senda Editorial, Madrid, Spain (1999).

12. C. AHNERT, J. M. ARAGONÉS, O. CABELLOS, and N. GARCÍA-HERRÁNZ, "Continuous Validation and Development for Extended Applications of the SEANAP Integrated 
3-D PWR Core Analysis System," Mathematics and Computation, Reactor Physics, and Environmental Analysis in $\mathrm{Nu}$ clear Applications, J. M. ARAGONÉS, Ed., Vol. 1, p. 710, Senda Editorial, Madrid, Spain (1999).

13. N. GARCÍA-HERRANZ, O. CABELlOS, J. M. ARAGONÉS, and C. AHNERT, “Analytic Coarse Mesh Finite Difference Method Generalized for Heterogeneous Multidimen- sional Two-Group Diffusion Calculations," Nucl. Sci. Eng., 144, 23 (2003).

14. A. GEIST, A. BEGUELIN, J. DONGARRA, R. MANCHEK, W. JAING, and V. SUNDERAM, PVM: A User's Guide and Tutorial for Networked Parallel Computing, MIT Press, Boston (1994).

José M. Aragonés [MS, industrial and energy engineering, 1969, and $\mathrm{PhD}$, nuclear engineering, 1977, the Polytechnic University of Madrid (UPM), Madrid, Spain] is the chair of nuclear physics in the Department of Nuclear Engineering of UPM and a member of the Nuclear Science Committee (NSC) of the Organization for Economic Cooperation and Development (OECD) Nuclear Energy Agency (NEA). He is chairman of the NEA/Nuclear Science Committee (NSC) Expert Group on light water reactor (LWR) transients. His background includes the development of three-dimensional (3-D) neutron-kinetics (NK) and thermalhydraulics $(\mathrm{TH})$ codes for coupled core analysis and their validation with plant operation data and with the OECD/NEA/NSC benchmarks on LWR transients.

Carol Ahnert (MS, nuclear physics, 1969, and PhD, physics, 1986, Central University of Madrid, Spain) is the chair of nuclear engineering in the Department of Nuclear Engineering of UPM. Her background includes the development and validation of 3-D NK and TH codes for coupled core analysis and fuel management.

Oscar Cabellos (MS, industrial and energy engineering, 1993, and $\mathrm{PhD}$, nuclear engineering, 1998, UPM, Spain) is an associate professor in nuclear physics in the Department of Nuclear Engineering of UPM. His background includes the development of neutronics lattice codes and the coupling of NK and TH codes.

Nuria García-Herranz (MS, industrial and energy engineering, 1995, and $\mathrm{PhD}$, nuclear engineering, 1999, UPM) is an assistant professor in energy engineering in the Department of Energy Engineering of the Open University of Madrid. Her background includes the development of advanced 3-D neutronics nodal codes and the validation of NK and TH coupled codes.

Vanessa Aragonés-Ahnert (MS, industrial and energy engineering, UPM, 1997) is engineer analyst for qualified consumers of the Spanish open electric market in the ENDESA Energy Company. Her background includes the validation of NK and TH coupled codes with the OECD/NEA/NSC benchmarks on LWR transients. 